\title{
A fault model of the 1995 Kobe earthquake derived from the GPS data on the Akashi Kaikyo Bridge and other datasets
}

\author{
Kazuki Koketsu, Shingo Yoshida, and Hiromichi Higashihara \\ Earthquake Research Institute, University of Tokyo, Bunkyo-ku, Tokyo 113-0032, Japan
}

(Received February 8, 1998; Revised July 7, 1998; Accepted August 3, 1998)

\begin{abstract}
Co-seismic horizontal displacements, which have been obtained from recently released GPS observations on the Akashi Kaikyo Bridge, are examined for their consistency with displacements observed in the vicinity of the bridge. An E18.7 $\mathrm{S}$ displacement of $25.0 \mathrm{~cm}$ should be removed from them. The adjusted data indicate an additional fault segment beneath Akashi Strait. We construct a new fault model by adding this segment to a model assumed previously. We then recover the slip distribution over the new model by inverting these data together with other geodetic observations. The displacements calculated from the recovered distribution fit the observations well, and the distribution of slip indicates that the additional segment is closely related to the southern main segment of the previous model. Joint inversion of the geodetic and waveform datasets suggests large slips with longer duration in the shallow parts of these segments.
\end{abstract}

\section{Introduction}

The Kobe (Hyogo-ken Nanbu) earthquake (January 17, 1995 JST; $M_{\mathrm{W}}$ 6.9) caused Kobe and its neighboring cities the worst damage in the Japanese modern history since the 1923 Kanto earthquake ( $\left.M_{\mathrm{S}} 8.2\right)$. This attracted much attention from seismologists, and many models have since been derived for its source fault system.

There are three kinds of datasets available: 1 . geodetic data (Hashimoto et al., 1996; Tabei et al., 1996), 2. teleseismic waveforms provided by Incorporated Research Institutions for Seismology, and 3. strong motion seismograms (Japan Meteorological Agency (JMA), 1995; Toki et al., 1995; Kobe city government). Hashimoto et al. (1996) derived a fault model from dataset 1. Kikuchi and Kanamori (1996) determined the fault rupture process by using dataset 2 . Sekiguchi et al. (1996) and Ide et al. (1996) obtained the slip history over their fault models from dataset 3. Horikawa et al. (1996) simultaneously inverted datasets 2 and 3 for the slip history. Yoshida et al. (1996) and Wald (1996) carried out joint inversions of the three datasets.

Most of these studies adopted similar assumptions for the fault geometry based on the distribution of aftershocks (Hirata et al., 1996; Katao et al., 1997) and the pattern of geodetic displacements. The source fault system is assumed to consist of southern and northern segments. They run along the Nojima fault on Awaji island, and the Suma, Suwayama, and Gosukebashi faults in the Kobe area, which have been specified as active faults by the Research Group for Active Faults of Japan (RGAFJ, 1991). For example, Fig. 1 shows the fault geometry adopted by Yoshida et al. (1996) and the epicenter of the main shock located by Katao et al. (1997). The Akashi Strait separates the two rupture segments $A$ and

Copy right (C) The Society of Geomagnetism and Earth, Planetary and Space Sciences (SGEPSS); The Seismological Society of Japan; The Volcanological Society of Japan; The Geodetic Society of Japan; The Japanese Society for Planetary Sciences.
$B$, which are nearly vertical with slight dips (dip angles are $75^{\circ}$ and $85^{\circ}$ ) in opposite directions to each other. Katao et al. (1997) also determined the focal mechanism in Fig. 1 from first $\mathrm{P}$ motions, and this indicates right-lateral strike faulting.

The Akashi Kaikyo Bridge was being constructed over Akashi Strait to connect Awaji island with Kobe City (Fig. 1). This is now a three-span suspension bridge consisting of two tower pillars (2P and 3P in Fig. 2) and two anchorages (1A and 4A). Co-seismic displacements due to the Kobe earthquake have been observed by GPS (global positioning system) instruments installed on the bridge, and were presented by Yamagata et al. (1996, arrows with dotted legs in Fig. 2). Yamagata et al. (1996) reported that no evidence of slide or subsidence was observed by sight at $1 \mathrm{~A}$ and $4 \mathrm{~A}$ on the ground. The foundations of $2 \mathrm{P}$ and $3 \mathrm{P}$ were inspected by various devices, and no evidence was observed by any of them except a sliding micrometer at $2 \mathrm{P}$ and a borehole camera close to 3P. The former indicated $20 \mathrm{~mm}$ subsidence, and the latter observed $35 \mathrm{~mm}$ displacement of a mud layer at a depth of $4 \mathrm{~m}$, but no displacement was observed in deeper basements, which bear the foundation of 3P. Therefore, the horizontal displacements by GPS on the bridge are expected to contain no independent movement of the bridge simply representing the co-seismic crustal movement itself.

The pattern of most horizontal displacements in Fig. 2 indicates right-lateral strike-slip along the two fault segments like the focal mechanism, but $3 \mathrm{P}$ and $4 \mathrm{~A}$ were displaced westward against $1 \mathrm{~A}$ and $2 \mathrm{P}$ in disagreement with the pattern. This suggests that another fault segment runs across the bridge between $2 \mathrm{P}$ and $3 \mathrm{P}$, but it does not coincide with the Nojima fault, the Suma fault, or their extensions, so that none of the previous studies considered it. We will here examine the possibility of this new segment and derive a more accurate fault model by using the GPS data on the bridge and the other datasets. 


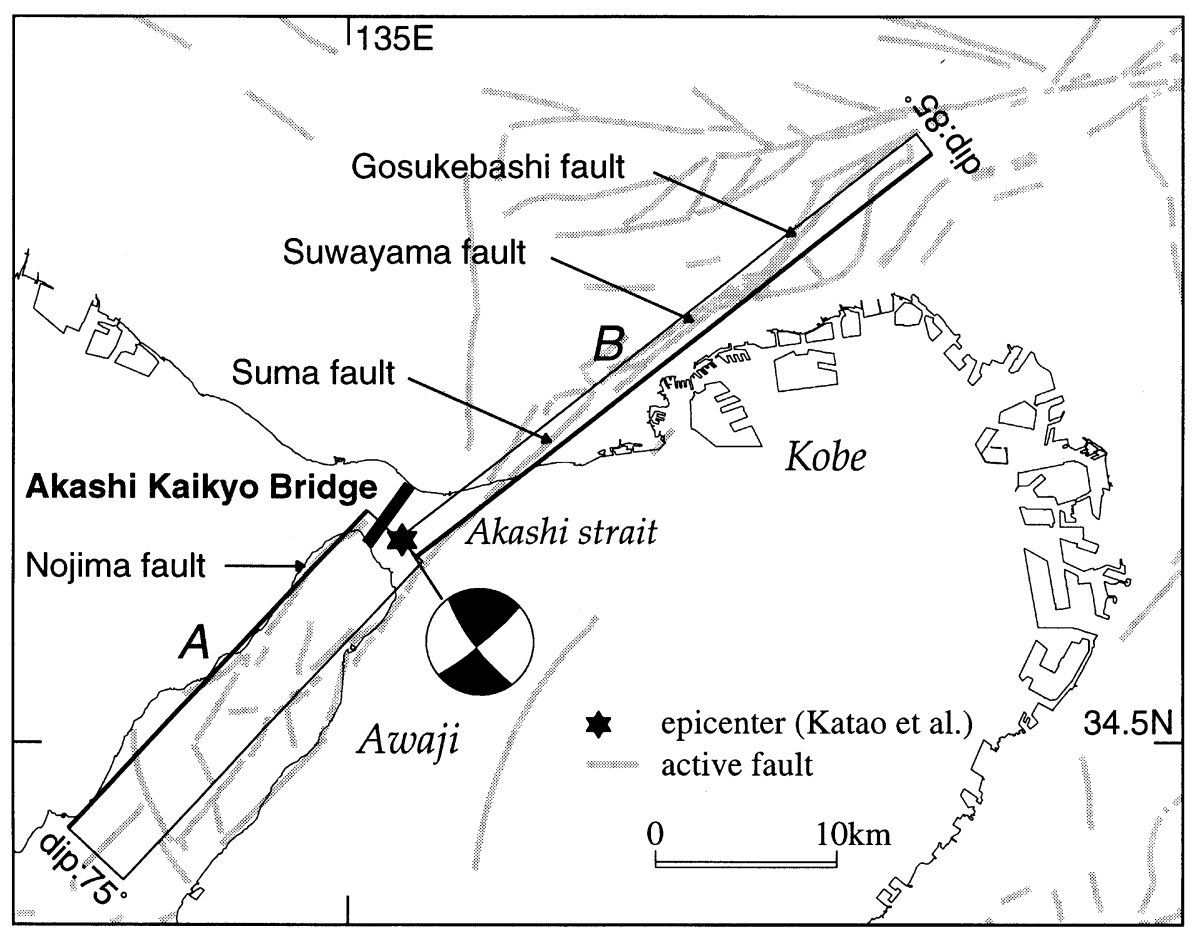

Fig. 1. Akashi Kaikyo Bridge (black bar) and the fault model of Yoshida et al. (1996) for the 1995 Kobe earthquake. Two rectangles represent the surface projections of the fault segments and thick lines indicate their shallower sides. The solid star with a focal mechanism solution is the epicenter of the main shock determined by Katao et al. (1997), and gray lines are active fault traces specified by RGAFJ (1991).

\section{Geodetic Dataset}

Two institutions and one consortium have carried out geodetic surveys in the Kobe-Awaji area before and after the 1995 Kobe earthquake. First of all, the Geographical Survey Institute of Japan (GSI) conducted three surveys in the KobeAwaji area at its primary or secondary control points:

(1) triangulation survey in 1886-1903,

(2) EDM (electro-optical distance meter) survey in 1977-1985,

(3) GPS survey immediately after the earthquake in 1995.

Hashimoto et al. (1996) derived an inner coordinate solution for horizontal displacements of the primary control points during the period from 1977-1985 to 1995 , by using the results of surveys (2) and (3). This solution is shown by arrows with open heads in Fig. 2. As are indicated by $3 \sigma$ confidence ellipses, the solution has only very small errors. GSI also carried out leveling along the routes L1 and L2 of Fig. 2 in 1970-1990 or 1994, and immediately after the earthquake. Hashimoto et al. (1996) derived vertical displacements of benchmarks during this period by fixing the western and eastern ends of L1 and the southern end of L2 (upper and right panels in Fig. 2).

Secondly, the Japanese University Consortium for GPS Research (JUNCO) conducted GPS surveys at several sites in 1989-1994 and immediately after the earthquake. Tabei et al. (1996) derived horizontal and vertical displacements from the results of these surveys by fixing the coordinates of a survey site in Kyoto, and an IGS global tracking site in Usuda. Since both the sites are far from the Kobe-Awaji area, and Hashimoto et al. (1996) derived displacements smaller than $1 \mathrm{~cm}$ around them, we can expect no serious error due to the assumption of the fixed points. The horizontal displacements are shown by arrows with gray heads in the map of Fig. 2, and the vertical ones are shown by small squares in the panel on the upper-right corner of Fig. 2. Observational errors, which are indicated by $3 \sigma$ confidence ellipses and $\sigma$ bar for horizontal and vertical displacements, respectively, are sufficiently small except for those of the horizontal and vertical displacements at TSUN and MIDO.

Thirdly, the Honshu-Shikoku Bridge Authority (HSBA, 1995 ) conducted GPS surveys before and immediately after the earthquake at the four sites on the bridge. These surveys were extended to the two secondary GSI control points TK-2 and No. 34 along approaching roads to the bridge. Yamagata et al. (1996) analyzed their results together with those of GSI surveys (1) and (3) (Sagiya, personal communication), and obtained horizontal displacements during the period from 1886-1903 to 1995, which are indicated by arrows with solid heads and dotted legs in Fig. 2. Since survey (1) was carried out about hundred years ago, it is less appropriate for deriving co-seismic displacements than survey (2). However, survey (2) did not cover secondary control points, and so Yamagata et al. (1996) had to use the result of survey (1). The HSBA survey before the earthquake was conducted at earliest in 1988 , but it was probably assumed to be done at the same time as survey (1).

Accordingly, the displacements derived by Yamagata et al. (1996) include long-term eastward motion of the KobeAwaji area (Iimura et al., 1997) for the period between surveys (1) and (2). The comparison of horizontal displacements of Hashimoto et al. (1996, arrows with open heads in 

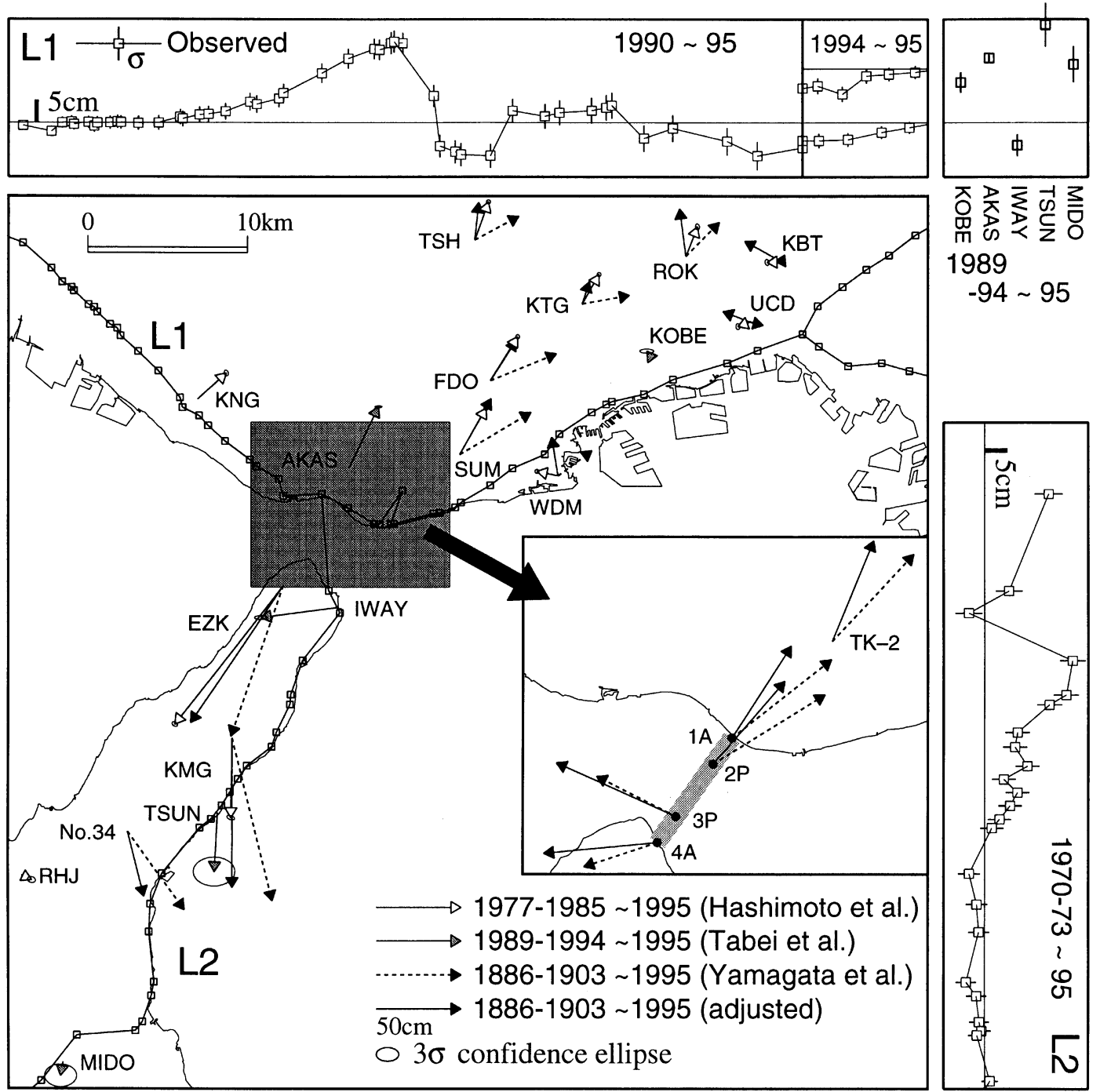

$-94 \sim 95$

Fig. 2. Geodetic dataset in the vicinity of the source region. Arrows with open or gray heads indicate horizontal co-seismic displacements obtained by Hashimoto et al. (1996) and Tabei et al. (1996). Arrows with solid heads and dotted legs indicate those used by Yamagata et al. (1996), and they have been translated to arrows with solid legs by minimizing their differences from the open arrows. The panels outside the map show vertical displacements derived from leveling (Hashimoto et al., 1996) and GPS observations (Tabei et al., 1996). Three- $\sigma$ confidence ellipses and one- $\sigma$ error bars are attached to horizontal and vertical displacements, respectively.

Fig. 2) and Yamagata et al. (1996, arrows with solid heads and dotted legs) shows that this translation motion is obvious at sites close to the bridge and source faults, and as large as about $30 \mathrm{~cm}$ (Hashimoto, 1998). We determine the motion to be $25.0 \mathrm{~cm}$ in the direction of $\mathrm{E} 18.7^{\circ} \mathrm{S}$ by minimizing the sum of squares of the difference between the two sets of displacements. This least-squares adjustment reduces the RMS (root-mean-squares) difference from $25.9 \mathrm{~cm}$ to $6.6 \mathrm{~cm}$. A "difference" or "residual" of two displacements is defined here as the length of a vector drawn from one to the other.

If the eastward motion is then removed from the displacements of Yamagata et al. (1996), the adjusted displacements (arrows with solid heads and legs in Fig. 2) agree well to those of Hashimoto et al. (1996) at sites such as EZK, SUM, FDO, KTG and TSH. Therefore a new geodetic dataset, shown in Fig. 3 with open symbols, is built by combining the adjusted displacements on the bridge with the horizontal and vertical displacements by Hashimoto et al. (1996) and Tabei et al. (1996).

\section{Previous Fault Model}

Most of the horizontal displacements (arrows with open heads) in Fig. 3 still point to the south-west or north-east indicating right-lateral strike faulting, and the northern segment $B$ of the fault model adopted by Yoshida et al. (1996) agrees well with the boundary of the regions of these two motions. This model will be called YSO hereafter. In addition, we find only upheaval in the western part of the L1 leveling route from the crossing over $B$, while subsidence is mostly observed in the eastern part. This would suggest that the faulting of $B$ includes some west-up dip slips. On the other hand, upheaval is obtained at almost all points along route L2 on Awaji island. Since the southern segment $A$ extends along the western coast of the island, this segment should be associated with east-up dip slips. These features of the faulting are recovered well in the slip distribution derived by 

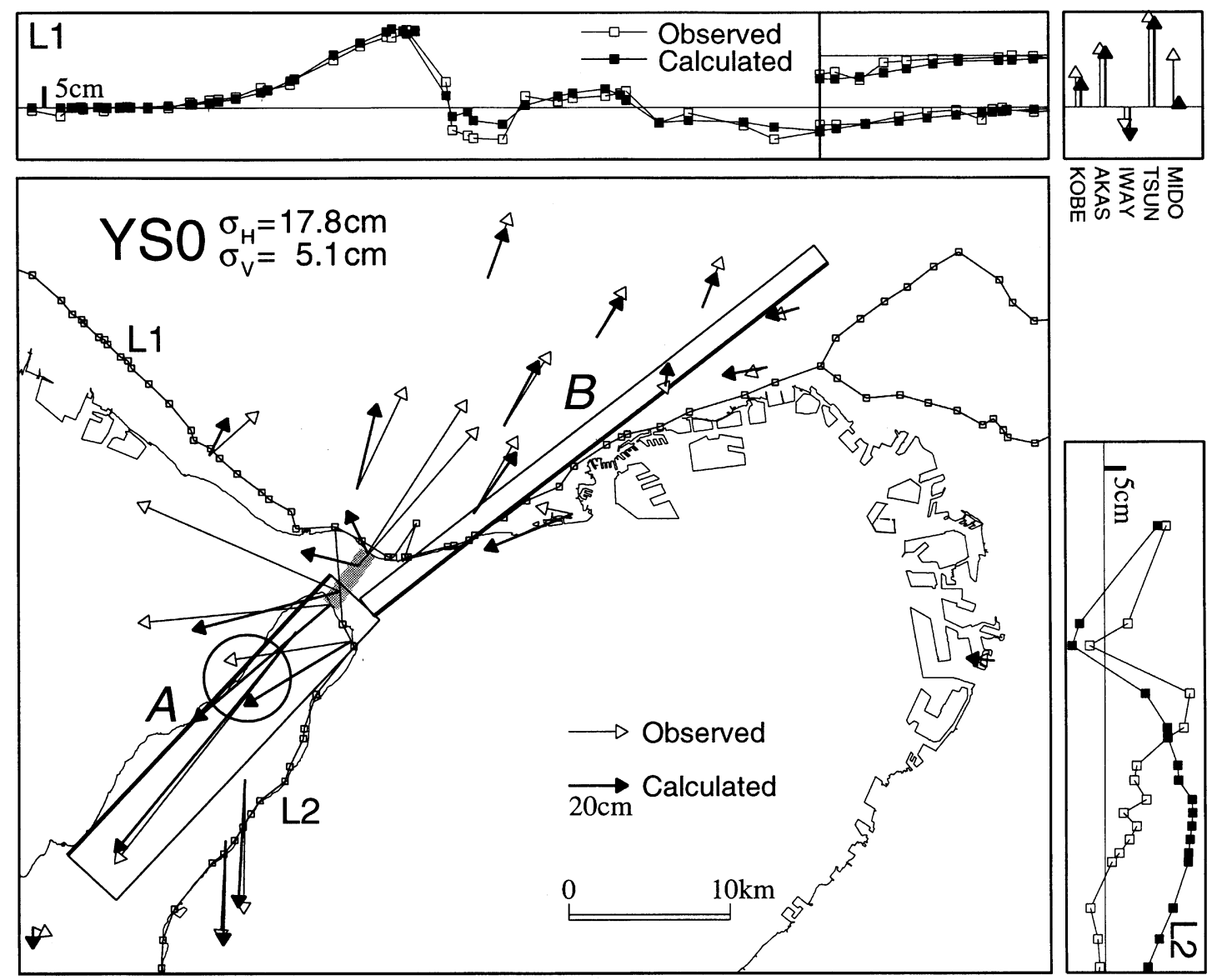

Fig. 3. Displacements calculated for the fault model YS0 (solid symbols) in comparison with those observed (open symbols).

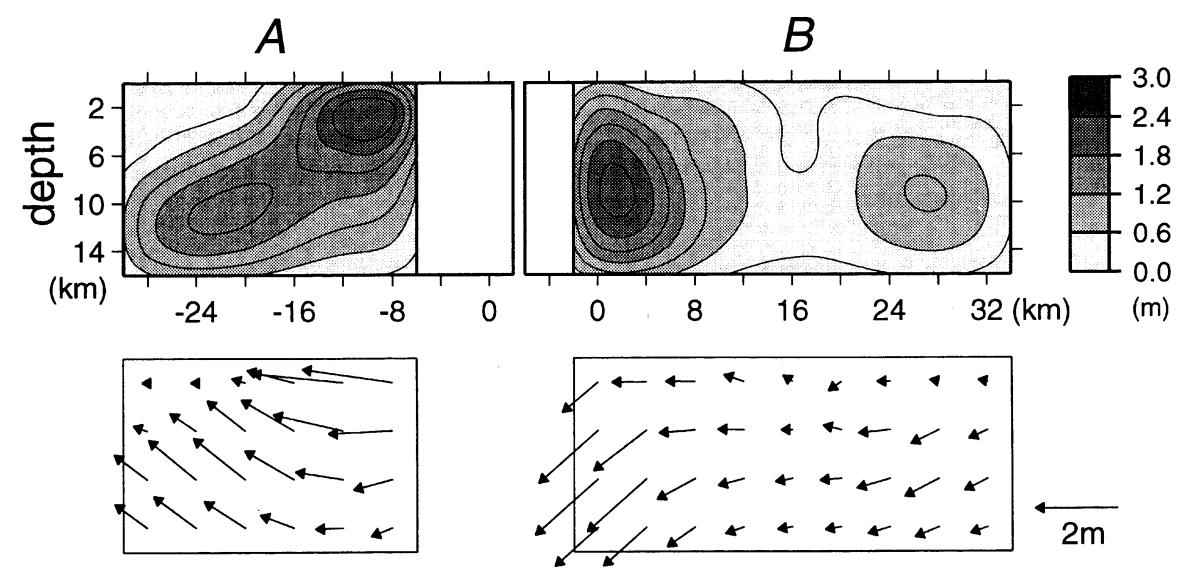

Fig. 4. Slip distribution over the fault model YS0 derived by Yoshida et al. (1996) through inversion of a geodetic dataset. Arrows in the lower panel represent the slip vectors on the eastern walls of the fault segments, and their magnitudes are shown in the upper contour diagram.

Yoshida et al. (1996) as shown in Fig. 4 (note that the arrows in the figure show the movement of the eastern walls). The distribution also indicates that large slips over $2 \mathrm{~m}$ happened in the northern-shallow part of $A$ and the southern-deep part of $B$. The former coincides with the area under the surface trace of the Nojima fault (see Fig. 1).

In the southern-deep part of $A$, Yoshida et al. (1996) re- covered rather large up-dip slips, which are due to the large uplifts obtained by Tabei et al. (1996) from GPS measurement at TSUN and MIDO. However, these uplifts are actually associated with large errors as shown in Fig. 2, and the result of leveling along L2 indicates much smaller displacements around there. In addition, Tabei et al. (1996) themselves mentioned that vertical displacements derived from 

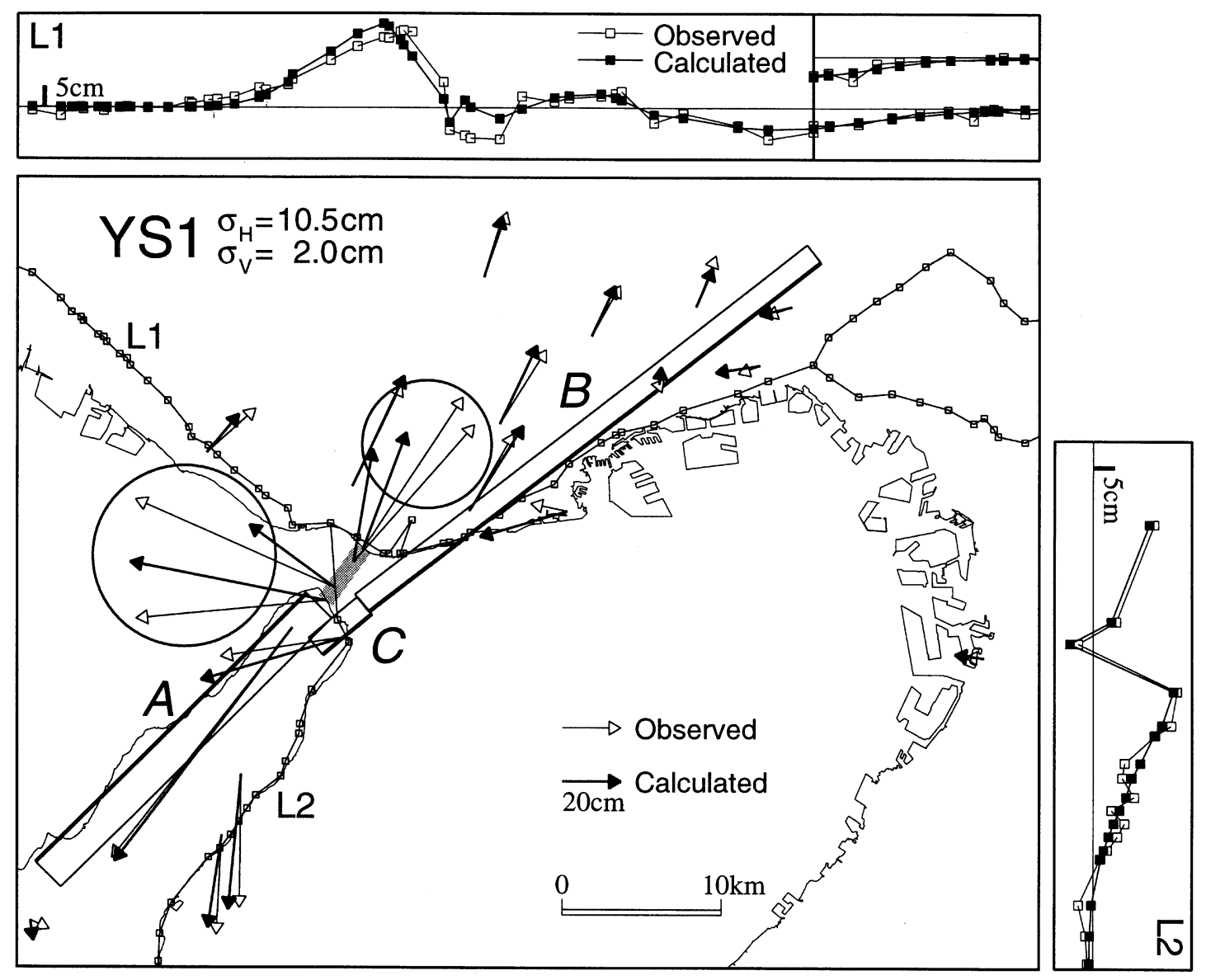

Fig. 5. The fault model YS1 consists of segments $A$ and $B$, and the southward extension of $B$, which is denoted by $C$. Displacements calculated from the inversion for this model (solid symbols) are shown in comparison with those observed (open symbols).

GPS measurements are generally less accurate than horizontal ones, so we will not use their vertical displacements in later analyses.

Since the data on the bridge and along the leveling route L2 were released late, Yoshida et al. (1996) could not include them in their analysis. Consequently, the synthetic displacements (solid symbols in Fig. 3) calculated from the slip distribution in Fig. 4 do not agree with them at all, so it is necessary to revise the fault model. The RMS residual for all the observed and calculated displacements including them is 17.8 or $5.1 \mathrm{~cm}$ for the horizontal or vertical component, respectively. Here we note also that the observed westward displacement at IWAY is inconsistent with the fault model described above, and that the calculated horizontal displacement in Fig. 3 does not agree with this observation, as shown in a large circle of Fig. 3 .

\section{New Fault Models}

Horikawa et al. (1996) already showed that an extension of the northern segment to Awaji island, together with a steeper dip for the southern segment, could correct the contradiction at IWAY. We therefore construct model YS1 by adding extension $C$ to segment $B$ of YS0, and giving a dip of $82^{\circ}$ to segment $A$ as shown in Fig. 5. These modifications result in a step-over between the northern and southern segments at the northern part of Awaji island, and Akashi Strait should be a pull-apart basin due to this fault system.

We then invert the geodetic dataset for a slip distribution on model YS1. Except for the different dataset and fault geometry, we adopt the same method (least-squares with smoothing and positivity constraints; see Yoshida and Koketsu (1990) for details) and statistical parameters as those used by Yoshida et al. (1996) for YS0. For example, the segments are divided into $4 \times 4 \mathrm{~km}$ pieces, each of which is represented by a point dislocation at its center. The weight of smoothing constraint relative to data residuals is determined by minimizing ABIC (Akaike's Bayesian Information Criterion). Figure 5 also compares the observed displacements (open symbols) with the ones calculated from the inversion result (solid symbols). The horizontal and vertical RMS residuals are reduced by half to 10.5 and $2.0 \mathrm{~cm}$, but those of the displacements at the four sites on the bridge, which are highlighted in the two large circles, are as large as 23.7, 36.9, 41.8 and $20.2 \mathrm{~cm}$, though satisfactory agreement is obtained for all the other data including the horizontal displacement at IWAY.

The westward displacements at 3P and 4A of the bridge can be explained if another right-lateral segment extends west to east in the north of 3P. We accordingly introduce a diagonal segment connecting $A$ and $B$ under Akashi Strait, and remove $C$ and the undersea part of $B$ to avoid fault branching. There are too few aftershocks around there to determine the dip 

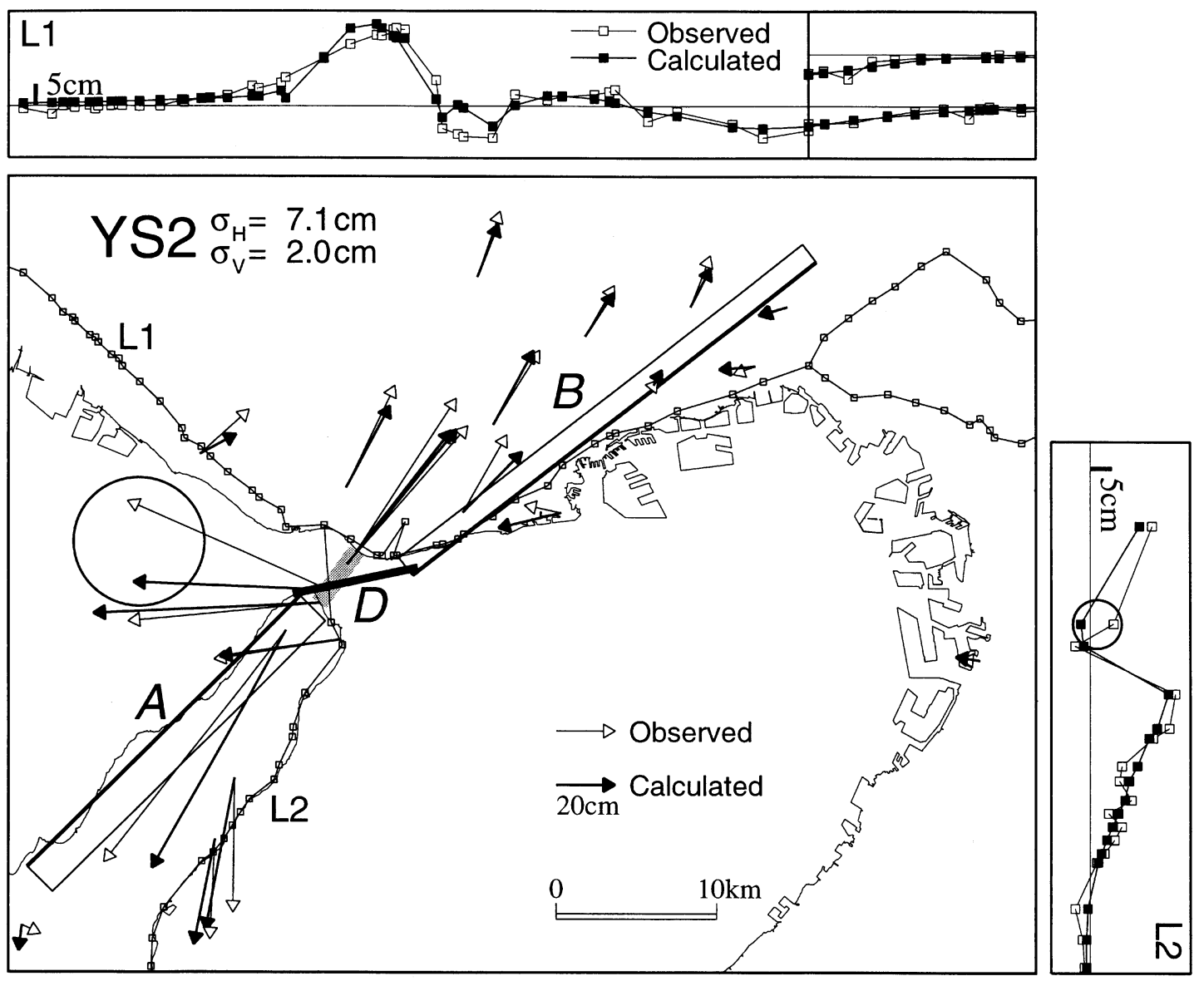

Fig. 6. The fault model YS2 consists of segment $A$, segment $B$ without its underwater part, and the diagonal segment $D$ connecting them. Displacements calculated from the inversion for this model (solid symbols) are shown in comparison with those observed (open symbols).

of this diagonal segment $D$, so we simply assume it to be vertical by taking an approximate average of dips of $82^{\circ} \mathrm{E}$ and $85^{\circ} \mathrm{W}$ for the segments $A$ and $B$.

We again carry out an inversion for this model YS2, and show the synthetic displacements in Fig. 6 compared with the observation. The horizontal RMS residual is reduced to $7.1 \mathrm{~cm}$, and we can find improvement on the bridge, but the contradiction at 3P is still as large as $29.0 \mathrm{~cm}$. In addition, as indicated by a circle in the right small panel of Fig. 6, subsidence now appears along the leveling route L2 at the northern tip of Awaji island, where upheaval was observed.

\section{Final Fault Model}

In order to remove the above contradictions, we finally constructed model YS3 by merging YS1 and YS2. This model includes the diagonal segment $D$ as well as the undersea part of $B$ and its southward extension $C$. The synthetic displacements calculated from the inversion for this model agree well with the observations, as shown in Fig. 7. The horizontal and vertical RMS residuals are further reduced to 6.4 and $1.7 \mathrm{~cm}$, respectively.

The derived slips in Fig. 8 again indicate right-lateral faulting and $A$ has some dip-slip components of east-up, while $B$ and $C$ have some of west-up. Compared to the slip distribution for model YS0 in Fig. 4 , the $1 \sim 1.5 \mathrm{~m}$ slips in the southern-deep part of $A$ have been removed by the in- troduction of the data along L2 and the removal of vertical displacements by Tabei et al. (1996) as mentioned in Section 3. As a result, the asperity ("asperity" meaning here just a zone of large slips) in the northern-shallow part is intensified as far as $2.4 \mathrm{~m}$. This asperity $a_{1}$ is extended to the western part of $D$. On the other hand, the asperity $a_{2}$ in the southern-deep part of $B$ is somewhat weakened due to its extension to $C$. Since the slip in the deep part of $D$ is small, and no significant down-dip components are derived in the whole of $D, D$ is naturally related to $A$ and disconnected from $B$ at its eastern part, where only very small slips are recovered.

We have been using the geodetic dataset alone, but we now jointly invert the strong motion seismograms and teleseismic waveforms with this dataset to investigate how the revision of the fault geometry affects the dynamic features of the fault system. We again adopt the same method and parameters as described in Yoshida et al. (1996). Comparing the result of this inversion (upper panel of Fig. 9) with Fig. 8, we find that asperity $a_{1}$ further extends to $D$ and is somewhat concentrated to the shallower part. Asperity $a_{2}$ is also concentrated in the deeper part, and its peak moves about $6 \mathrm{~km}$ to the north. In addition, the weak asperity at a distance of $26 \mathrm{~km}$ from the hypocenter (white star) in Fig. 8 is shifted $6 \mathrm{~km}$ to the south and developed as large as $1.3 \mathrm{~m}\left(a_{3}\right.$ in the upper panel of Fig. 9). Except for the extension to $D$, these fea- 

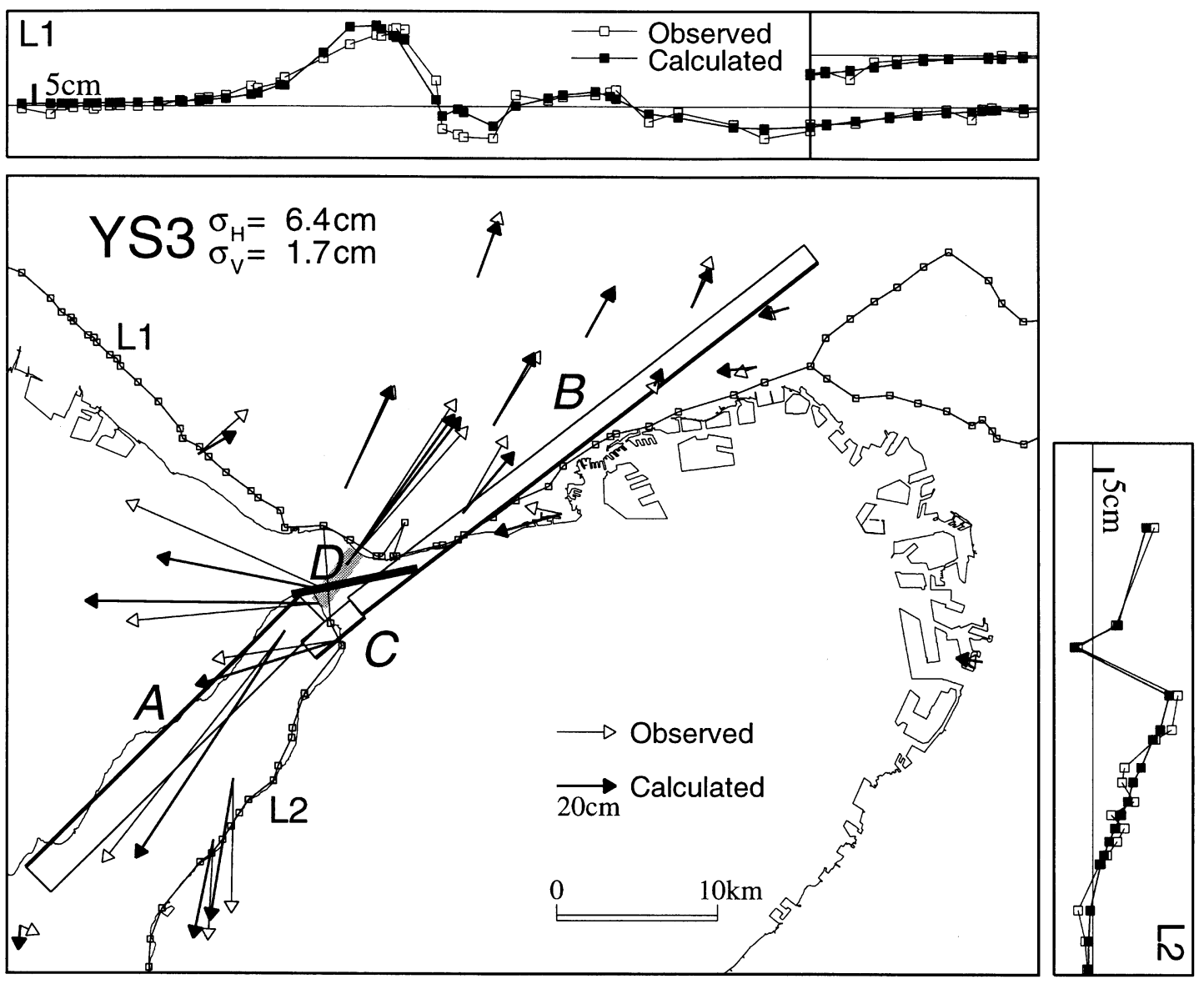

Fig. 7. The fault model YS3 is constructed by merging models YS1 and YS2. Displacements calculated from the inversion for this model (solid symbols) are also shown in comparison with those observed (open symbols).

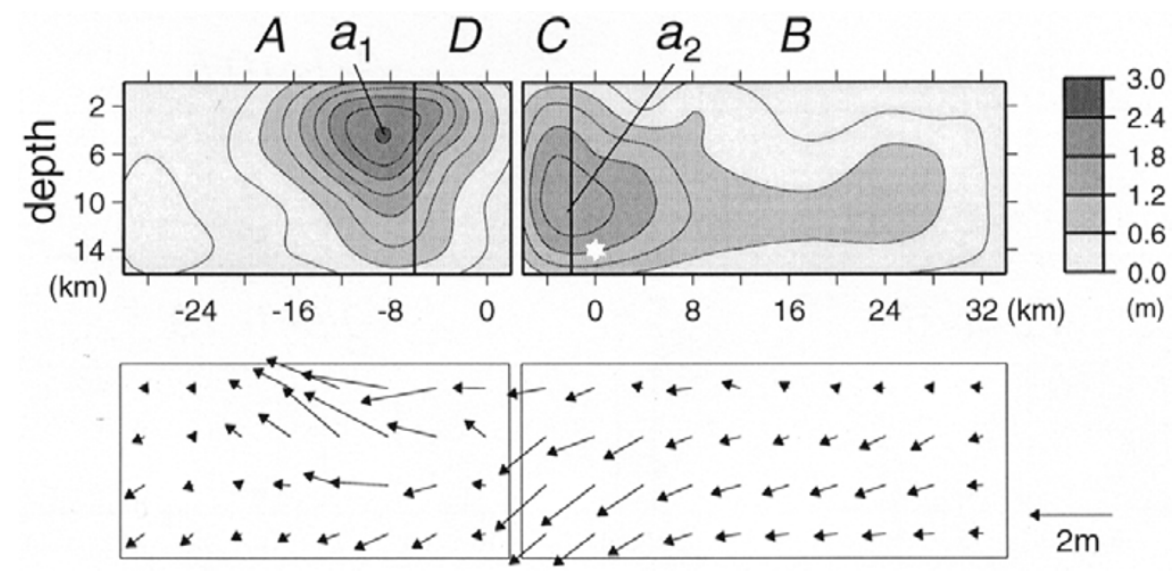

Fig. 8. Slip distribution over the fault model YS3 derived by inversion of the geodetic dataset. Arrows in the lower panel represent the slip vectors on the eastern walls of the fault segments, and their magnitudes are shown in the upper contour diagram. $a_{1}$ and $a_{2}$ indicate "asperities" (areas of large slip), and the white star denotes the hypocenter.

tures have already appeared in Yoshida et al.'s (1996) result for model YS0 (lower panel of Fig. 9). However, asperity $a_{1}$ of model YS3 is more developed, including larger dip-slip components of east-up, and the slips of $0.6 \sim 1.2 \mathrm{~m}$ above $a_{2}$ are relocated to the shallow part of $C$.
Finally, Fig. 10 represents the time-dependent features of the fault system YS3 by showing the slip distribution extracted from Fig. 9 at $1.5 \mathrm{~s}$ intervals. The main fault rupture starts from the hypocenter indicated by a white or black star, and propagates bilaterally to the south and north. The 


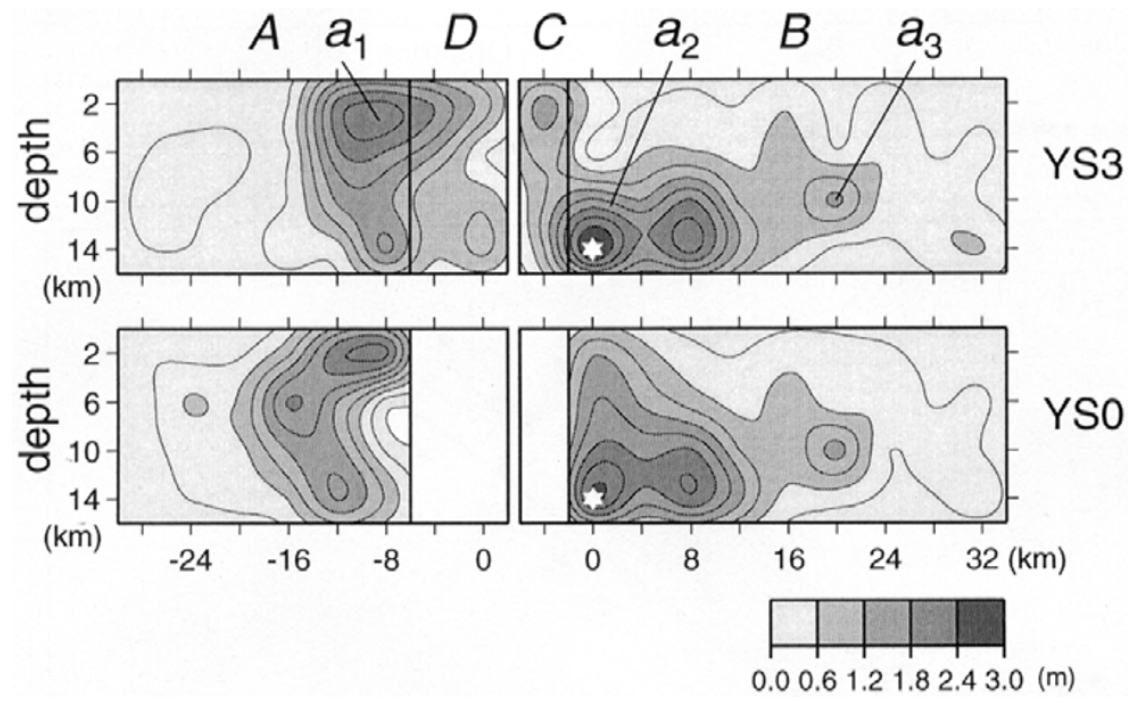

Fig. 9. Slip distribution over the fault model YS3 derived by joint inversion of the geodetic and waveform datasets (upper). The result of Yoshida et al. (1996) for model YS0 is also shown in the lower panel. $a_{1}, a_{2}$ and $a_{3}$ indicate "asperities" (areas of large slip), and the white star denotes the hypocenter.
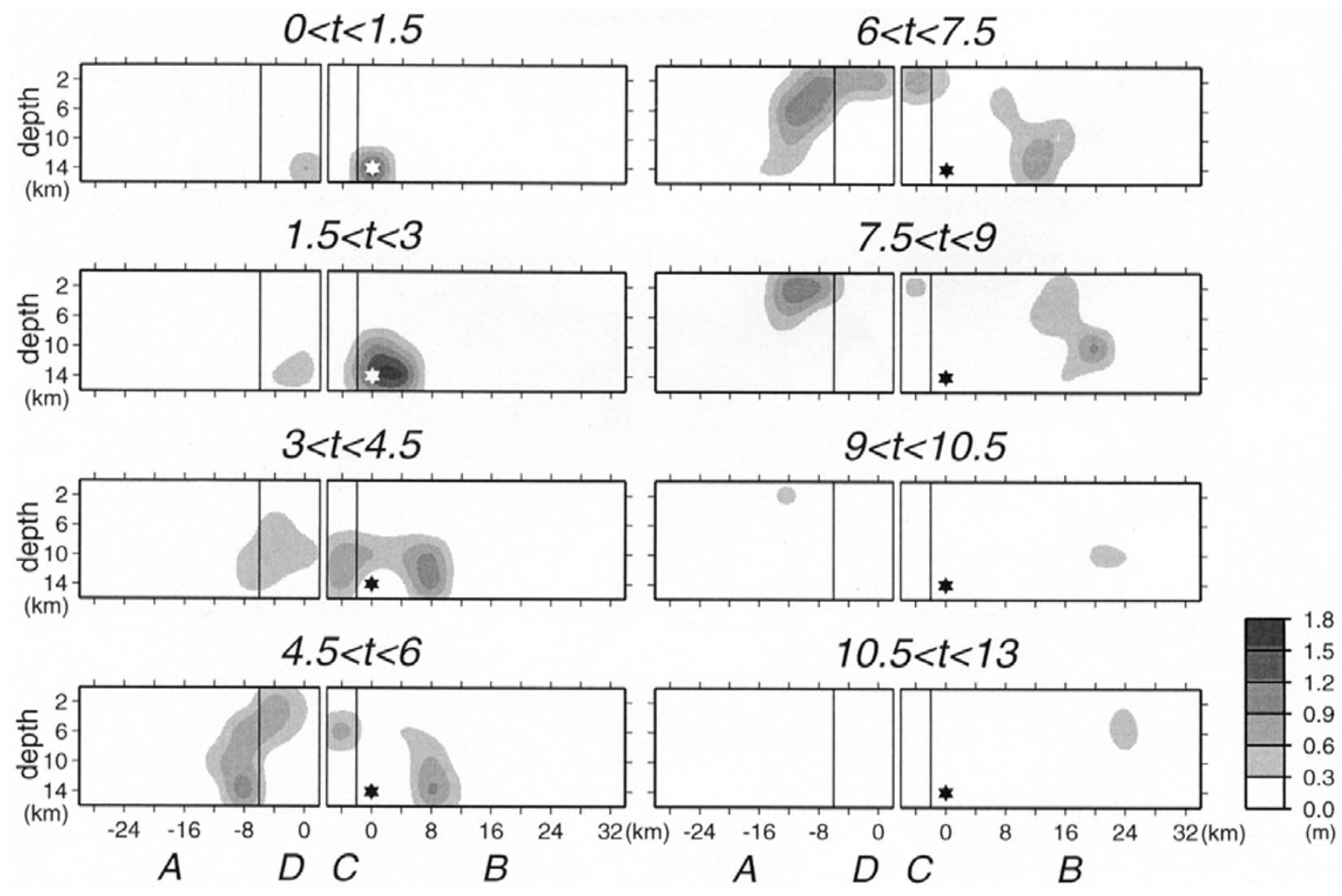

Fig. 10. Slip distributions extracted from the result of the joint inversion for model YS3 at every $1.5 \mathrm{~s}$. White and black stars denote the hypocenter.

southern rupture stays at the shallow parts of $A$ and $D$ for a relatively long time, while the northern rupture runs with an almost constant velocity. Another weak rupture propagates mostly upward from the northern bottom corner to the shallow part of $C$. This and the southern rupture almost simultaneously reach the shallow parts of $C, D$ and the northern
$A$ during the period of $6 \mathrm{~s}<t<7.5 \mathrm{~s}$, so that a large area is slipping at that time.

\section{Conclusion and Discussion}

(1) The horizontal displacements obtained from GPS observations at the Akashi Kaikyo Bridge should be adjusted 
to the result of Hashimoto et al. (1996) by removing an E18. $7^{\circ} \mathrm{S}$ displacement of $25.0 \mathrm{~cm}$.

(2) The fault system of the 1995 Kobe earthquake was previously assumed to consist of two segments separated from each other at Akashi Strait, but the adjusted displacements require an additional segment traversing the strait diagonally and connecting the main segments.

(3) The slip distribution derived from the displacements and other data shows that the additional segment has similar characteristics to those of the southern main segment.

(4) The result of the joint inversion of geodetic and waveform datasets indicates that the shallow part of the additional segment and southern main one form a large asperity of slips with longer duration.

We also note that the pull-apart basin does not extend along the main fault segments, but perpendicularly to them forming Akashi Strait. This is a morphological evidence for the additional fault segment (Bilham and King, 1989). However, no foreshock or aftershock has been found under Akashi Strait with focal mechanism expected from this additional segment, though some linearments parallel to the segment were observed by sonic reflections and boring surveys before the earthquake (Yamagata et al., 1996).

Acknowledgments. We are grateful to the Geographical Survey Institute of Japan and Honshu-Shikoku Bridge Authority for providing us the geodetic data. In particular, Takeshi Sagiya of GSI kindly found the displacements presented to HSBA in piles of documents.

A thorough review by Simon Williams, and constructive comments by Simon Williams, Manabu Hashimoto, Kosuke Heki and Neil Frazer were very helpful for improving the manuscript. This study was completed during the first author's visit to the School of Ocean and Earth Science Technology, University of Hawaii at Manoa. We thank Neil Frazer and Evelyn Norris for their kind arrangements.

\section{References}

Bilham, R. and G. King, The morphology of strike-slip faults: Examples from the San Andreas fault, California, J. Geophys. Res., 94, 10204 10216, 1989.

Hashimoto, M., Source process based on geodetic data, in Report on the Hanshin-Awaji Earthquake Disaster, Common Series Vol. 2, pp. 123139, Maruzen, Tokyo, 1998 (in Japanese with English abstract).

Hashimoto, M., T. Sagiya, H. Tsuiji, Y. Hatanaka, and T. Tada, Co-seismic displacements of the 1995 Kobe earthquake, J. Phys. Earth, 44, 255-279, 1996.

Hirata, N., S. Ohmi, S. Sakai, K. Katsumata, S. Matsumoto, T. Takanami, A. Yamamoto, T. Nishimura, T. Iidaka, T. Urabe, M. Sekine, T. Ooida,
F. Yamazaki, H. Katao, Y. Umeda, K. Ito, M. Nakamura, N. Seto, T. Matsushima, H. Shimizu, and Japanese University Group for the Urgent Joint Observation for the 1995 Hyogo-ken Nanbu Earthquake, Urgent joint observation of aftershocks of the 1995 Hyogo-ken Nanbu earthquake, J. Phys. Earth, 44, 317-328, 1996.

Honshu-Shikoku Bridge Authority, Report of the Effects of the Hyogoken Nanbu Earthquake on the Akashi Kaikyo Bridge, 41pp., 1995 (in Japanese).

Horikawa, H., K. Hirahara, Y. Umeda, M. Hashimoto, and F. Kusano, Simultaneous inversion of geodetic and strong motion data for the source process of the Hyogo-ken Nanbu, Japan, earthquake, J. Phys. Earth, 44, 455-471, 1996.

Ide, S., M. Takeo, and Y. Yoshida, Source process of the 1995 Kobe earthquake: Determination of spacio-temporal distribution by Bayesian modeling, Bull. Seismol. Soc. Am., 86, 547-566, 1996.

Iimura, Y., S. Miyazaki, and M. Sasaki, Establishment of new dense GSI's nationwide GPS array, Kokudochiriin Jiho, 87, 37-49, 1997 (in Japanese).

Japan Meteorological Agency (JMA), The 1995 Hyogoken-Nanbu earthquake and its aftershocks, Rep. Coord. Comm. Earthq. Pred., 54, 584592, 1995 (in Japanese).

Katao, H., N. Maeda, Y. Hiramatsu, Y. Iio, and S. Nakao, Detailed mapping of focal mechanisms in/around the 1995 Hyogo-ken Nanbu earthquake rupture zone, J. Phys. Earth, 45, 105-119, 1997.

Kikuchi, M. and H. Kanamori, Rupture process of the Kobe, Japan earthquake of Jan. 17, 1995 determined from teleseismic body waves, J. Phys. Earth, 44, 429-436, 1996.

Research Group for Active Faults of Japan (RGAFJ), Active Faults in Japan, revised edition, 437pp., University of Tokyo Press, Tokyo, 1991 (in Japanese with English abstract).

Sekiguchi, H., K. Irikura, T. Iwata, Y. Kakehi, and M. Hoshiba, Minute locating of fault planes and source process of the 1995 Hyogo-ken Nanbu (Kobe), Japan, earthquake from the waveform inversion of strong ground motion, J. Phys. Earth, 44, 473-487, 1996.

Tabei, T., T. Kato, J. P. L. Catane, T. Chachin, K. Fujimori, K. Hirahara, A. Kubo, T. Matsushima, T. Nakano, S. Nakao, S. Otsuka, T. Terashima, and T. Yamamoto, Crustal deformation associated with the 1995 Hyogoken Nanbu earthquake, Japan derived from GPS measurements, J. Phys. Earth, 44, 281-286, 1996.

Toki, K., K. Irikura, and T. Kagawa, Strong motion records in the source area of the Hyogoken-nambu earthquake, January 17, 1995, Japan, J. Nat. Disast. Sci., 16, 23-30, 1995.

Wald, D. J., Slip history of the 1995 Kobe, Japan, earthquake determined from strong motion, teleseismic and geodetic data, J. Phys. Earth, 44, 489-503, 1996.

Yamagata, M., M. Yasuda, A. Nitta, and S. Yamamoto, Effects on the Akashi Kaikyo bridge, Soils and Foundations, special issue, 179-187, 1996.

Yoshida, S. and K. Koketsu, Simultaneous inversion of waveform and geodetic data for the rupture process of the 1984 Naganoken-seibu, Japan, earthquake, Geophys. J. Int., 103, 355-362, 1990.

Yoshida, S., K. Koketsu, B. Shibazaki, T. Sagiya, T. Kato, and Y. Yoshida, Joint inversion of the near- and far-field waveforms and geodetic data for the rupture process of the 1995 Kobe earthquake, J. Phys. Earth, 44, 437-454, 1996.

K. Koketsu (e-mail: inquiry@taro.eri.u-tokyo.ac.jp), S. Yoshida, and H. Higashihara 